Meta

Journal des traducteurs

Translators' Journal

\title{
Une banque commerciale? Ça dépend...
}

\section{Carlos Del Burgo}

Volume 24, numéro 2, juin 1979

URI : https://id.erudit.org/iderudit/002508ar

DOI : https://doi.org/10.7202/002508ar

Aller au sommaire du numéro

Éditeur(s)

Les Presses de l'Université de Montréal

ISSN

0026-0452 (imprimé)

1492-1421 (numérique)

Découvrir la revue

Citer cet article

Del Burgo, C. (1979). Une banque commerciale? Ça dépend... Meta, 24(2),

273-276. https://doi.org/10.7202/002508ar d'utilisation que vous pouvez consulter en ligne.

https://apropos.erudit.org/fr/usagers/politique-dutilisation/ 


\section{UNE BANQUE COMMERCIALE ? ÇA DEPEND...}

Savoir différencier les divers types de banques est capital. En effet, nulle confusion ne saurait être tolérée dans un secteur qui touche d'aussi près le consommateur et le monde financier et bancaire. Et donc le monde des traducteurs. Pourtant, l'usage, en bon monarque, a des caprices qu'on peut difficilement ignorer. Ainsi, nous nous trouvons aux prises avec des termes plus ou moins synonymiques, tels que chartered bank, banque à charte, banque commerciale, banque, et de façon plus sporadique, banque de commerce ${ }^{1}$, banque privilégiée ${ }^{2}$, banque accréditée ${ }^{3}$, banque d'affaires ${ }^{4}$, banque de dépôt, banque populaire, com-

1. J.V. Servotte, Dictionnaire commercial et financier, Anvers (Belgique), Scriptoria, et Verviers (Belgique), Les Nouvelles Editions Marabout, 1978, 500 p. plus annexes, s.v. banque.

2. G. Dagenais, Dictionnaire des difficultés de la langue française au Canada, Montréal, Editions Pedagogia, 1967, XV +679 p., s.v. banqque.

3. Néologisme.

4. En France. Signalons la trilogie des banques d'affaires, banques de dépôt et établissements financiers, qui sont régis par les lois françaises et non les lois canadiennes. A noter également, la Banque commerciale de Paris, qui appartient à la catégorie des banques d'affaires. 
mercial bank ${ }^{5}$, trade bank, commercial trade bank, bank of commerce ${ }^{6}$, deposit bank, joint-stock bank, clearing bank, members bank, credit bank ${ }^{7}$, etc.

Il semble cependant que cet abus de termes correspondants (mais pas équivalents) constitue un facteur de babélisme au lieu d'être un outil facilitant la compréhension des activités bancaires déjà complexes en elles-mêmes.

Nous n'étudierons ici que certains termes dont l'utilisation est répandue au Canada ou dont le bon usage rendra plus aisée la communication entre locuteurs d'une même langue, et moins risquée la traduction des concepts en question, à savoir chartered bank, commercial bank, banque, banque commerciale et banque d charte.

La première différence à établir entre chartered bank et commercial bank est d'ordre géographique, ces termes étant usités respectivement au Canada et aux Étas-Unis. L'autre nuance est certes plus déroutante. Il s'agit d'un flottement de sens, d'un usage qui n'est pas ou qui n'est plus fixé. En effet, s'il est sûr que la chartered bank, que nous connaissons tous, s'adresse au grand public comme aux entreprises et aux gouvernements, l'étiquette américaine commercial bank est bien plus polysémique. La définition qu'en donne Filkins ${ }^{8}$, confirmée par celles de Nemmers ${ }^{9}$ et de Clark ${ }^{10}$, en fait une institution dont les activités s'orientent nettement vers le financement d'entreprises privées ou publiques, alors que le Funk \& Wagnalls ${ }^{11}$, qui, de manière inexplicable, omet le syntagme chartered bank, est plus vague et laisse à penser que l'apport des consommateurs n'est nullement exclu.

Pour traduire commercial bank, il faut donc connaître la nature du système bancaire en question et tenir compte du critère de régionalisme et de l'imprécision des notions au sein d'une même communauté linguistique, aux U.S.A. Pour les synonymes de ce terme et les acceptions qu'ils prennent derrière le rideau de mer (en Angleterre), on se reportera à l'excellent Penguin Dictionary of Economics.

Par ailleurs, la terminologie française ne fait pas non plus l'unanimité. Si l'usage local favorise l'emploi de banque à charte, l'Office de la langue française ${ }^{12}$ traduit chartered bank par banque commerciale, qu'il définit comme suit :

5. W. Funk, Funk \& Wagnalls Standard College Dictionary (édition canadienne), Montréal, Fitzhenry \& Whiteside, 1976, XXVI + 1590 p., s.v. commercial bank.

6. H. Van Hoof, Terminologie économique/Economic Terminology, Münich, Hueber, et Paris, Dunod, 1967,771 p., p. 123.

7. G. Bannock, R.E. Baxter \& R. Rees, The Penguin Dictionary of Economics, Markham (Canada), Penguin Books, 1978,467 p., s.v. commercial banks.

8. An organization chartered by either the comptroller of the Currency or the state in which it is located to engage in the business of banking; that is, accept deposits subject to check and make short term loans to business enterprises $»$, in : J.H. Filkins et D.L. Caruth, Lexicon of American Business Terms, New York, Simon and Schuster, 1973, p. 24.

9. E.E. Nemmers, Dictionary of Economics and Business, Totowa (New Jersey), Littlefield, Adams \& Co., 1967, p. 78 , no 33.

10. D.T. Clark \& B.A. Gottfried, University Dictionary of Business and Finance, New York, Thomas Y. Crowell, 1967, p. 30.

11. W. Funk, déjà cité.

12. Office de la langue française, Vocabulaire de l'économie (deuxième édition provisoire), Québec, Editeur officiel du Québec, 1977, s.v. chartered bank. 
Banque constituée en vertu de la loi canadienne sur les banques, dont sont exclues les caisses populaires, les caisses d'épargne, les coopératives de crédit, etc.

Note : L'expression «banque à charte » est un calque de l'anglais ${ }^{13}$.

Il faut donc s'accorder sur le contenu sémantique du mot «banque ». Pour Bernard et Colli ${ }^{14}$, il s'agit d'une

entreprise qui fait profession habituelle de recevoir du public, sous forme de dépôts ou autrement, des fonds qu'elle emploie pour son propre compte en opérations d'escompte, en opérations de crédit ou en opérations financières.

$\mathbf{M}^{\text {me }}$ de Villers-Sidani ${ }^{15}$ préconise, quant à elle, la substitution, pour autant que le contexte s'y prête, de l'ellipse «banque » à l'expression banque commerciale. On trouve néanmoins dans le Dagenais ${ }^{16}$ les précisions suivantes :

(Les) banques, toutes, y compris celles qui ne sont pas privilégiées, ont nécessairement obtenu de l'Etat un acte constitutif, une charte, avant de commencer à exercer leur commerce. Ce qui distingue les chartered banks des autres, c'est qu'elles ont des droits que les autres banquiers n'ont pas. L'entreprise des Caisses populaires, par exemple, est à proprement parler une banque, mais ce n'est pas une banque privilégiée.

Ici, M. Marchand ${ }^{17}$ fait remarquer qu'au contraire, une banque est, par définition, une institution à but lucratif (ce qui exclut les caisses populaires) qui est admise à la compensation, offre un régime de crédit réglementé et est tenue de garder des réserves auprès de la Banque du Canada. Ceci ne correspond nullement à la situation actuelle de la banque d'épargne, qui devrait théoriquement porter le nom de caisse d'épargne.

Face à ces quatre définitions, il apparaît utile de contourner la difficulté. En ce qui a trait à nos onze chartered banks ${ }^{18}$ canadiennes ou aux commercial banks américaines, on peut éviter toute ambiguïté en adoptant l'une des trois solutions suivantes :

Canada

chartered bank

1. banque commerciale canadienne
U.S.A.

commercial bank

banque commerciale américaine

13. Dagenais dit que le terme de droit monarchique «charte " n'a plus cours en français international (voir observation no 2). Le terme anglais charter est, lui, tout à fait vivant. Ainsi, le Random House (édition de 1973) donne sous la rubrique charter les cooccurrents suivants : to charter a bank.

14. Y. Bernard \& J.-C. Colli, Vocabulaire économique et financier, Paris, Editions du Seuil (Collection Points-économie), 1976, s.v. banque.

15. Responsable de la terminologie de la gestion à l'Office de la langue française.

16. G. Dagenais, déjà cité.

17. Rédacteur à la Banque canadienne nationale.

18. Voici la liste de ces banques : la Banque de Montréal, la Banque de la Nouvelle-Ecosse, la Banque Toronto-Dominion, la Banque provinciale du Canada, la Banque de commerce canadienne impériale, la Banque royale du Canada, la Banque canadienne nationale, la Banque mercantile du Canada, la Banque de la Colombie-Britannique, la Norbanque et la Banque commerciale et industrielle du Canada. Le nombre de chartered banks sera bientôt porté à treize, avec l'arrivée de la Banque Continental I.A.C. (Industrial Acceptance Corporation) et de la Banque d'épargne de la cité et du district de Montréal. 
2. banque commerciale (chartered bank)

3. banque accréditée ${ }^{19}$ (néol.) banque privilégiée banque commerciale

(commercial bank)

banque commerciale

Il semble que la paire banque privilégiée/banque commerciale offre la meilleure maniabilité et la plus forte adéquation linguistique. Pour le critère de fréquence, c'est bien sûr l'avenir qui décidera.

Carlos S. Del Burgo 